\title{
Desmotivadora evolución de la desconexión asimétrica del Núcleo Accumbens en el trastorno por consumo de cocaína: un punto de vista traslacional
}

\author{
Demotivating outcome of asymmetrical \\ Nucleus Accumbens disconnection for cocaine \\ related disorder: a translational point of view
}

\author{
Gonzalo Haro*,**, Julia Renau-Lagranja***, Victor Costumero****, Abel BaQuero******, \\ Emilio Meneu******, John Salamone*******, Mercì Correa************. \\ * Research Group TXP, School of Medicine, Universidad Cardenal Herrera-CEU, CEU Universities, Castellón (Spain). \\ ** Dept. of Mental Health, Hospital Provincial de Castellón (Spain). *** Dept. of Internal Medicine, Hospital Comarcal \\ de Vinaròs, Vinaroz (Spain). **** Dept. of Basic Psychology and Psychobiology, Universitat Jaume I, Castellón (Spain). \\ ***** Amigó Foundation, Castellón (Spain). ****** Dept. of Neurology, Hospital La Plana, Villarreal (Spain). \\ ******** Behavioral Neuroscience Div., University of Connecticut, Storrs, CT (USA).
}

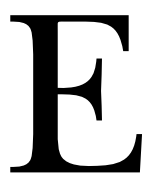

stimado editor,

La desconexión neuroanatómica utilizada en animales revela que el circuito cortico-límbico estriado que involucra a la corteza prefrontal medial (mPFC), la amígdala basolateral (BLA), y el núcleo accumbens (Nacb) media en los procesos de toma de decisiones (Salamone y Correa, 2012; Salamone, Yohn, LópezCruz, San Miguel y Correa, 2016). Las proyecciones BLA modulan la actividad del Nacb e influyen en la dirección del comportamiento hacia estímulos relevantes (Floresco, Blaha, Yang y Phillips, 2001). La interrupción de la actividad del Nacb y de la BLA, así como su comunicación, reduce la preferencia por opciones que implican más esfuerzo, aumenta la preferencia para opciones más arriesgadas, con un comportamiento menos motivado (Salamone y Correa, 2012; Salamone et al., 2016).

Con esta carta, los autores presentan la historia de un paciente después de una psicocirugía extensa, incluyendo la neuroimagen y la evaluación neuropsicológica y la discusión realizada desde un punto de vista traslacional.

El paciente fue adoptado pocos días después de nacer con distocia. Se desconoce la historia de la familia biológica. Sus padres adoptivos sufrieron un trastorno relacionado con la cocaína y el alcohol. Durante la infancia, el paciente tenía la mayoría de los síntomas del trastorno de déficit de atención con hiperactividad (TDAH). Fue descrito como un niño agresivo y tenía un diagnóstico de trastorno de conducta. A los 14 años, empezó el consumo de cocaína. A los 16 años, fue diagnosticado de "trastorno del control de impulsos no especificado" y trastorno relacionado con la cocaína. Su cociente de inteligencia era 85 . A los 17 años, un psiquiatra diagnostica al paciente con un "trastorno mental secundario a distocia". La imagen de resonancia magnética (MRI) indica atrofia del hemisferio izquierdo. La tomografía por emisión de positrones (TEP) muestra una reducción general moderada en el metabolismo cerebral. Basado en el último diagnóstico y el estudio de neuroimagen, un neurocirujano diagnosticó al paciente de "disfunción del control de impulsos" y de "síndrome de disfunción límbica”, prescribiendo psicocirugía para el control de la agresividad.

La neurocirugía consistió en lesiones de coagulación térmica inducidas por radiofrecuencia. Las regiones de destino eran la corteza cingulada anterior (AC), la cápsula anterior y la estría terminal de hemisferio izquierdo y la cápsula anterior y la amígdala del hemisferio derecho. Se realizó una segunda cirugía, extendiendo las lesiones en la cápsula anterior izquierda y en la AC derecha dos meses más tarde, en vista de una recaída en la agresividad.

Después de la psicocirugía, la agresividad persistía, y el paciente comenzó a tener delirios. El paciente alternó periodos de consumo de cocaína y heroína con períodos de permanencia en centros penitenciarios. Se le diagnosticó esquizofrenia paranoide sin adherencia al tratamiento. A la edad de 27 años, mostró déficits cognitivos predominantes y sintomatología psicótica negativa.

Recibido: Febrero 2018; Aceptado: Marzo 2018.

Enviar correspondencia a:

Gonzalo Haro, Universidad Cardenal Herrera-CEU. Calle Grecia, 31 - 12006 (Castellón) Spain.

E-mail: gonzalo.haro@uchceu.es. Tel. +34 964372402 

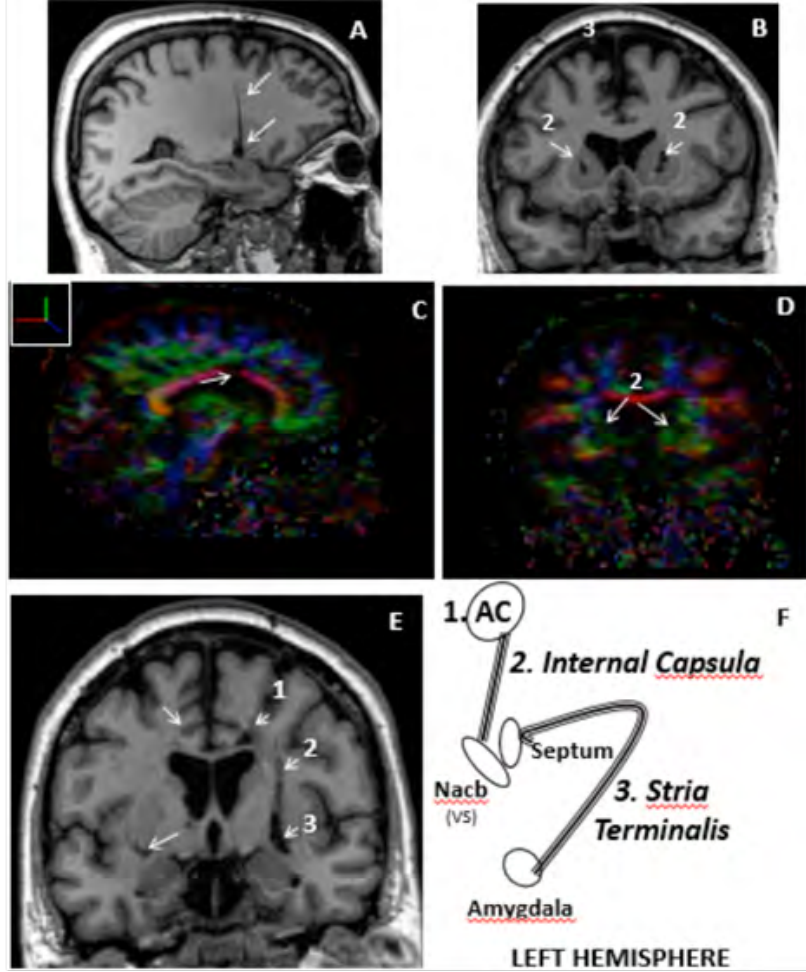

LEFT HEMISPHERE

Figura 1.

Prueba MRi realizada 15 años después de la cirugía (A, B y E). Imágenes de tractografía que muestran el impacto en el cuerpo calloso (C) y las fibras de la cápsula interna (D). (F) Esquema con las regiones de destino de la neurocirugía. La meta de la cirugía era desactivar la AC y desconectarla del estriado ventral

(VS) y de la amígdala en ambos hemosferios, y producir una lesión

de la amígdala del hemisferio derecho y desconectar las fibras eferentes de la amígdala en el hemisferio izquierdo.

Quince años después de la psicocirugía, a los 32 años, se realizó una exploración de neuroimagen más completa (Figura 1). La MRI mostró cavidades anormales en el putamen derecho, el Nacb izquierdo, la cápsula interna y las AC de ambos hemisferios. La tractografía mostró mayor impacto en las conexiones entre la AC, el Nacb y la amígdala. Los análisis morfológicos mostraron una marcada reducción en el volumen de las cortezas parietales y la PFC medial, incluyendo la corteza orbital y ventromedial. Además, la evaluación neuropsicológica (BIS-11, LSRP, IOWA Gambling Task y Tower of London [Torre de Londres]) mostró un deterioro de los procesos de planificación, dificultades en la toma de decisiones acerca del costo/beneficio, rasgos psicópatas y alta impulsividad motor.

Las lesiones combinadas unilaterales de la mPFC y el Nacb en hemisferios opuestos (desconexión) en animales redujo la selección de opciones que implican esfuerzo y produjo la perseverancia de comportamiento sin integrar la información de retroalimentación afectiva (Salamone y Correa, 2012; Hauber y Sommer, 2009). Por otra parte, la desconexión entre la mPFC y otras regiones del estriado (p.ej., el caudado-putamen medial ) deteriora aspectos de la función atencional y los recuerdos basados en la costumbre (Phillips y Carr, 1988; Packard, Hirsh y White, 1989). En los seres humanos, la conectividad funcional entre la AC y el Nacb, así como, entre la AC y la amígdala, es relevante para la toma de decisiones y la anticipación de la recompensa, respectivamente (Cohen, Heller y Ranganath, 2005; Marsh, Blair, Vythilingam, Busis y Blair, 2007). Además, las tareas no rutinarias, que requieren supervisión constante de nueva información para planificar respuestas adecuadas, son particularmente susceptibles a lesiones en la PFC. Se ha informado que el paciente tiene dificultades en la adquisición de nuevos hábitos y muestra dificultades para mantener la atención. Además, la evaluación neuropsicológica sugiere deterioro de los procesos de planificación y dificultades en la toma de decisiones de costo/beneficio. Por lo tanto, estos patrones de comportamiento son consistentes con las lesiones producidas durante la cirugía y el daño resultante observado en las imágenes recientes.

Barcia et al. (2007) y Leiphart y Valone-III (2010) reconocieron que las psicocirugías más frecuentes son la capsulotomía anterior, que se ha mostrado beneficiosa para la ansiedad generalizada y los trastornos obsesivo-compulsivos, y la cingulotomía anterior, que ha mostrado mejoras en la depresión bipolar y los trastornos esquizoafectivos. Sin embargo, las mejoras menos relevantes fueron obtenidas en las adicciones y la esquizofrenia, y hay evidencia de que la combinación de más de un objetivo cerebral puede empeorar los resultados.

En esta carta, le sugerimos que la "disfunción del control de impulsos" y el "síndrome de disfunción límbica" cuando el paciente era adolescente se hubieran podido definir más adecuadamente como TDAH, trastorno de conducta y relacionado con la cocaína. Estos trastornos tienen tratamientos no quirúrgicos, específicos y eficaces. Quince años después de la psicocirugía, el paciente desarrolló esquizofrenia, y su agresividad persiste. Además, el paciente presenta mayor preferencia por opciones más arriesgadas, una incapacidad de integrar la información relacionada con la retroalimentación afectiva (castigo o premio), dificultades en la adquisición de nuevos hábitos y dificultades para mantener la atención. Teniendo en cuenta los modelos animales, estos deterioros funcionales son en parte debido a la psicocirugía, lo cual sugiere un pronóstico irreversible y desmotivador.

\section{Conflictos de interés}

No existen conflictos de interés Esta investigación no recibió ninguna subvención específica de las agencias de financiación de los sectores público, comercial, o sin fines lucrativos. La publicación en Open Access ha sido financiada por la Fundación de investigación del Hospital Provincial de Castellón. Todos los autores contribuyeron igualmente. 


\section{Aspectos éticos}

El comité de ética del Consorcio del Hospital Provincial de Castellón aprobó el estudio y se obtuvo el consentimiento informado del tutor legal y del participante para la experimentación (neuroimágenes y pruebas neuropsicológicas) y la publicación.

\section{Referencias}

Barcia, J.A., Bertolín-Guillén, J.M., Barcia-González, J., Campos, J. y Hernández, M.E. (2007). Estado actual de la psicocirugía en España. Neurocirugía, 18, 301-311.

Cohen, M.X., Heller, A.S. y Ranganath. C. (2005). Functional connectivity with anterior cingulate and orbitofrontal cortices during decision-making. Brain Research. Cognitive Brain Research, 23, 61-70. doi:10.1016/j.cogbrainres.2005.01.010.

Floresco, S.B., Blaha, C.D., Yang, C.R. y Phillips, A.G. (2001). Dopamine D1 and NMDA receptors mediate potentiation of basolateral amygdala-evoked firing of nucleus accumbens neurons. The Journal of Neuroscience, 21, 6370-6376.

Hauber, W. y Sommer, S. (2009). Prefrontostriatal circuitry regulates effort-related decision making. Cerebral Cortex, 19, 2240-2247. doi:10.1093/cercor/bhn241.

Leiphart, J.W. y Valone-III, F.H. (2010). Stereotactic lesions for the treatment of psychiatric disorders. Journal of $\mathrm{Neu}$ rosurgery, 113, 1204-1211.

Marsh, A.A., Blair, K.S., Vythilingam, M., Busis, S. y Blair, R.J. (2007). Response options and expectations of reward in decision-making: the differential roles of dorsal and rostral anterior cingulate cortex. Neuroimage, 35, 979-988. doi:10.1016/j.neuroimage.2006.11.044.

Packard, M.G., Hirsh, R. y White, N.M. (1989). Differential effects of fornix and caudate nucleus lesions on two radial maze tasks: evidence for multiple memory systems. Journal of Neurosurgery, 9, 1465-1472.

Phillips, A.G. y Carr, G.D. (1988). Cognition and the basal ganglia: a possible substrate for procedural knowledge. The Canadian Journal of Neurological Sciences, 14, 381-385.

Salamone, J.D. y Correa. M. (2012). The mysterious motivational functions of mesolimbic dopamine. Neuron, 76, 470-485. doi:10.1016/j.neuron.2012.10.021.

Salamone, J.D., Yohn, S.E., López-Cruz, L., San Miguel, N. y Correa, M. (2016). Activational and effort-related aspects of motivation: neural mechanisms and implications for psychopathology. Brain, 139, 1325-1347. doi:10.1093/brain/aww050. 